\title{
Rapid progression of Barrett's esophagus into adenocarcinoma in a combined lung and kidney transplant recipient
}

\author{
Waleed Saleh, MD, Andre Duranceau, MD, Jocelyne Martin, MD, Nicolas Noiseux, MD, \\ Charles Poirier, MD, and Pasquale Ferraro, MD, Montreal, Quebec, Canada
}

The incidence of cancer in solid organ transplant recipients is significantly greater than that of the general population. This increase is related to the intensity and duration of the immunosuppressive therapy. We herein present a rare case in which Barrett's esophagus (BE) rapidly progressed into invasive adenocarcinoma after a patient with scleroderma underwent combined lung and kidney transplantation.

\section{CLINICAL SUMMARY}

A 56-year-old male patient with scleroderma and pulmonary fibrosis (IPF) was referred to our lung transplant program. The patient presented with progressive dyspnea over a 2-year period. While on treatment, his condition deteriorated progressively until he became oxygen dependent.

The pretransplant workup revealed a forced expiratory volume in 1 second of $53 \%$, forced vital capacity of $47 \%$, and diffusing capacity for carbon monoxide of $13 \%$. Computed tomography (CT) scan showed pulmonary infiltrates consistent with IPF. An upper gastrointestinal endoscopy showed the presence of BE at $29 \mathrm{~cm}$. Biopsies confirmed BE with metaplasia but no evidence of dysplasia. The renal function was moderately impaired with a serum creatinine of 141 and a creatinine clearance of $36 \mathrm{~mL} / \mathrm{min}$. The patient had a thorough evaluation by the transplant nephrologists, who confirmed the renal insufficiency. The patient was thus listed for a combined double lung and kidney transplantation. While on the waiting list, the chest CT scan was repeated 3 times at 6-month intervals and remained unremarkable.

After a 21-month wait, the recipient underwent a sequential double-lung transplant on cardiopulmonary bypass. Once stabilized hemodynamically, a heterotopic kidney transplant was performed.

Postoperatively, immunosuppression consisted of basiliximab for induction and methylprednisolone/prednisone, tacrolimus, and mycophenolate mofetil as maintenance therapy. After being extubated on postoperative day 4, the patient developed infiltrates in both lungs and consolidation

\footnotetext{
From the Lung Transplantation Program, Division of Thoracic Surgery, Centre Hospitalier de l'Universite de Montreal, Montreal, Quebec, Canada.

Received for publication March 19, 2008; revisions received April 28, 2008; accepted for publication May 16, 2008; available ahead of print Sept 16, 2008.

Address for reprints: Pasquale Ferraro, MD, Division of Thoracic Surgery, Notre

Dame Hospital, Suite D-8051, 1560 Sherbrooke Street East, Montreal, Quebec,

Canada H2L 4M1 (E-mail: pasquale.ferraro@umontreal.ca).

J Thorac Cardiovasc Surg 2010;139:217-8

0022-5223/\$36.00

Copyright $₫ 2010$ by The American Association for Thoracic Surgery

doi:10.1016/j.jtcvs.2008.05.049
}

of his left lower lobe, and he became ventilator dependent. On postoperative day 14, a tracheostomy was carried out and the patient was then successfully weaned from the ventilator. Seven weeks postoperatively, blood was found in the nasogastric tube. Endoscopy revealed a large mass at the distal esophagus, which on biopsy was confirmed to be a poorly differentiated adenocarcinoma. CT scan showed an 8-cm mass of the distal esophagus (Figure 1). Due to the patient's overall condition and the apparent aggressiveness of the esophageal cancer, only comfort measures were instituted. The patient died 8 weeks after his transplantation.

\section{DISCUSSION}

The incidence of cancer in transplant recipients is increased secondary to the immunosuppressive therapy. This is especially noteworthy in patients with premalignant conditions. Reports have shown that in renal transplant recipients, the incidence of skin tumors is increased 30-fold, ${ }^{1}$ and posttransplant lymphoproliferative disorder predominates in lung transplant recipients, most likely as a result of the more intense immunosuppression. ${ }^{2}$

$\mathrm{BE}$ is a well-documented premalignant lesion. Endoscopic surveillance is generally recommended every 18 to 24 months, with follow-up at shorter intervals if there is a concern for dysplasia on histology. Once high-grade dysplasia develops, patients should be considered for esophagectomy, unless they are poor surgical candidates.

Immunosuppression may play an important role in the malignant transformation of $\mathrm{BE}$ into adenocarcinoma as

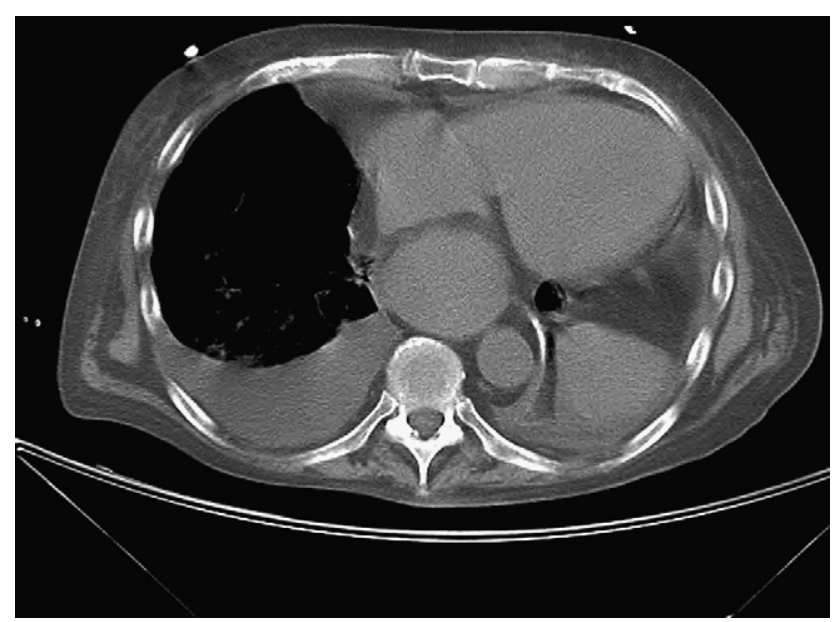

FIGURE 1. Computed tomography scan at the level of the lower chest showing large mass arising from the distal esophagus. 
suggested by Oka and colleagues. ${ }^{3}$ The authors found that patients with BE have significant suppression of T-cell and B-cell function as well as interlukin-2 production. In addition, Hsu and associates ${ }^{4}$ reported a case of metastatic esophageal cancer arising in $\mathrm{BE}$ in a patient with chronic lymphocytic leukemia with documented B-cell dysfunction. In the present report, the use of induction therapy followed by intense immunosuppression certainly placed the BE at increased risk for malignant transformation.

It is possible that the metaplasia in this patient may have progressed into high-grade dysplasia prior to the transplantation, taking into consideration the 22-month gap between the diagnosis of metaplasia and the transplant. Endoscopic surveillance during the waiting period would have permitted an early diagnosis; however, this was not possible in view of the patient's poor pulmonary status.

Only one case of rapid progression of BE to adenocarcinoma has been reported after liver transplantation, ${ }^{5}$ and to our knowledge, the present case is the first reported after lung transplantation.
It becomes essential to ask whether the diagnosis of $\mathrm{BE}$ in pretransplant patients precludes them from being transplanted. Although there is no clear answer, there is no doubt that these patients should undergo upper gastrointestinal endoscopy on a regular basis prior to their transplant. Close surveillance after transplantation is certainly warranted to detect signs of dysplasia. Should high-grade dysplasia occur, adjustment of the immunosuppressive therapy is necessary and ablation or resection of the dysplastic mucosa should be considered.

\section{References}

1. Andrés A. Cancer incidence after immunosuppressive treatment following kidney transplantation: a review. Crit Rev Oncol Hematol. 2005;56:71-85.

2. Ippoliti G, Rinaldi M, Pellegrini C, Vigano M. Incidence of cancer after immunosuppressive treatment for heart transplantation: a review. Crit Rev Oncol Hematol. 2005;56:101-13.

3. Oka M, Attwood SE, Kaul B, Smyrk TC, DeMeester TR. Immunosuppression in patients with Barrett's esophagus. Surgery. 1992;112:11-7.

4. Hsu CW, Krevsky B, Sigman LM, Thomas RM. Rapid progression of Barrett's esophagus to metastatic esophageal carcinoma in a patient with chronic lymphocytic leukemia. J Clin Gastroenterol. 1998;27:261-4.

5. Ilan Y, Shouval D, Galun E, et al. Esophageal malignancy after liver transplantation in a patient with Barrett's esophagus. Scand J Gastroenterol. 1996;31:415-6.

\title{
Nonobstructive ectopic accessory mitral valve tissue in association with left ventricular apical aneurysm
}

\author{
Lokeswara Rao Sajja, MS, MCh, MD, FACS, and Gopichand Mannam, FRCS (Glasg), FRCS(Ed), FRCS \\ (CT), Hyderabad, India
}

Accessory mitral valve tissue is a rare congenital cardiac anomaly, an uncommon cause of left ventricular outflow tract (LVOT) obstruction, and usually diagnosed during childhood. We report a case of a nonobstructive accessory mitral valve in a 33-year-old woman who was evaluated for systemic embolization that resulted in right hemiplegia. A mass lesion was diagnosed in the apical region of the left ventricular (LV) cavity by means of transthoracic and transesophageal echocardiography. A cardiac magnetic resonance image (MRI) showed a mass lesion in the LV cavity and LV apical aneurysm. The patient had undergone suc-

From the Lung Transplantation Program, Division of Thoracic Surgery, Centre Hospitalier de l'Universite de Montreal, Montreal, Quebec, Canada.

Received for publication April 9, 2008; revisions received April 24, 2008; accepted for publication May 20, 2008; available ahead of print Sept 16, 2008.

Address for reprints: Gopichand Mannam, FRCS (Glasg), FRCS(Ed), FRCS (CT), Department of Cardiothoracic Surgery, CARE Hospital, the Institute of Medical Sciences, Road No.1 Banjara Hills, Hyderabad, Andhra Pradesh 500034, India (E-mail: gopi.mannam@gmail.com).

J Thorac Cardiovasc Surg 2010;139:218-20

$0022-5223 / \$ 36.00$

Copyright (c) 2010 by The American Association for Thoracic Surgery doi:10.1016/j.jtcvs.2008.05.057 cessful excision of the mass lesion, which was accessory mitral valve tissue, and linear repair of the LV aneurysm.

\section{CLINICAL SUMMARY}

A 33-year-old woman who recovered from right hemiplegia was referred to our center with a diagnosis of an intracavitory mass lesion of the left ventricle. On physical examination, she had a regular pulse, with a rate of 80 beats/min and a blood pressure of $126 / 80 \mathrm{~mm} \mathrm{Hg}$. Her first and second heart sounds were normal, and there was no murmur. An electrocardiogram showed sinus rhythm. She was evaluated with transthoracic and transesophageal 2-dimensional echocardiography and Doppler scanning, which showed a mass in the LV cavity, and there was no gradient across the LVOT. A cardiac MRI demonstrated a mass lesion in the LV cavity attached to the distal interventricular septum and LV apical aneurysm (Figure 1, A). A coronary angiogram showed normal epicardial coronary arteries.

She underwent excision of accessory mitral valve tissue and linear repair of the LV aneurysm through a standard median sternotomy during cardiopulmonary bypass. The 